\title{
Nanocrystalline diamond surfaces for adhesion and growth of primary neurons, conflicting results and rational explanation
}

\section{Silviya M. Ojovan ${ }^{1}$, Mathew McDonald ${ }^{2}$, Noha Rabieh ${ }^{1}$, Nava Shmuel ${ }^{1,3}$, Hadas Erez', Milos Nesladek ${ }^{2}$ and Micha E. Spira ${ }^{1,3}$ *}

\footnotetext{
${ }^{1}$ Department of Neurobiology, The Alexander Silberman Institute of Life Science, The Hebrew University of Jerusalem, Jerusalem, Israel

${ }_{2}^{2}$ Institute for Materials Research in MicroElectronics - Interuniversity Micro Electronics Centre, Hasselt University, Antwerp, Belgium

${ }^{3}$ The Harvy M. Kruger Family Center for Nanoscience, The Hebrew University of Jerusalem, Jerusalem, Israel
}

\author{
Edited by: \\ Laura Ballerini, University of Trieste, \\ Italy \\ Reviewed by: \\ Alexander Dityatev, DZNE, Germany \\ Fabio Benfenati, University of Genoa, \\ Italy \\ *Correspondence: \\ Micha E. Spira, Department of \\ Neurobiology, The Alexander \\ Silberman Institute of Life Science \\ and The Harvy M. Kruger Family \\ Center for Nanoscience, The Hebrew \\ University of Jerusalem, Edmond J. \\ Safra Campus, Jerusalem 91904, \\ Israel \\ e-mail:spira@mail.huji.ac.il
}

Using a variety of proliferating cell types, it was shown that the surface of nanocrystalline diamond (NCD) provides a permissive substrate for cell adhesion and development without the need of complex chemical functionalization prior to cell seeding. In an extensive series of experiments we found that, unlike proliferating cells, post-mitotic primary neurons do not adhere to bare NCD surfaces when cultured in defined medium. These observations raise questions on the potential use of bare NCD as an interfacing layer for neuronal devices. Nevertheless, we also found that classical chemical functionalization methods render the "hostile" bare NCD surfaces with adhesive properties that match those of classically functionalized substrates used extensively in biomedical research and applications. Based on the results, we propose a mechanism that accounts for the conflicting results; which on one hand claim that un-functionalized NCD provides a permissive substrate for cell adhesion and growth, while other reports demonstrate the opposite.

Keywords: nanocrystalline diamonds, cultured neurons, poly-d-lysine, cell adhesion, calcium imaging, network connectivity

\section{INTRODUCTION}

The construction of efficient brain-machine interfaces (BMI) relies, to a large extent, on the use of biocompatible materials that can withstand the harsh biological solutions comprising the environment in which living cells operate. A promising substrate for such BMIs is the family of nanocrystalline diamond (NCD) which is a continuous layer of nanoscopic diamond crystals embedded in a nanoscale matrix of sp2 and disordered carbon (1-10\%; Daenen et al., 2009). NCD exhibits chemical and biochemical inertness, high corrosion resistance, excellent mechano-optical properties and large surface area (Fendrych etal., 2010). While intrinsic NCD is electrically insulating, it can be doped with boron to form semiconducting or even metallically conducting films with tunable surface chemistry (Singh et al., 2010; Zivcova et al., 2013). These properties make NCD and Boron-Doped NCD (BNCD) a very attractive material for electrodes or electrode coatings for the development of implantable electrodes for the restoration of sensory functions such as in cochlear or retinal implants (Xiao et al., 2006; Hadjinicolaou et al., 2012; Ganesan etal., 2014), to electrically communicate between neurons or muscles and peripheral prosthesis (Ariano et al., 2009) and for deep brain stimulation and recordings.

A major concern for using NCD coatings for multi-electrode arrays (MEAs) is whether it can serve as a permissive surface for post mitotic neuron adhesion and regenerative regrowth.

The general impression from the literature is that NCD substrates have superior biocompatible and adhesion permissive properties. Thus, it was reported that proliferating cells, including fibroblast, epithelial cells, and various cell lines, adhere and develop on bare (chemically non-functionalized) NCD surfaces. For example, Amaral etal. (2009) demonstrated that fibroblast cell line L929 (a mouse permanent cell line) and human gingival fibroblast adhere and proliferate on NCD surfaces without hydrophilic treatment or surface functionalization. Klauser et al. (2010), revealed that epithelial cells attach within $24-72 \mathrm{~h}$ onto oxygen-terminated NCD substrates without further surface functionalization and without the addition of fetal bovine serum (FBS) to the growth medium. Using the GT1-7 proliferating cell line, which was used to represent neurons, Ariano et al. (2009) documented that these cells adhere to $\mathrm{H}$ - or O-terminated NCD substrates without surface functionalization. In an apparent consistency with the above observations, Thalhammer et al. (2010) reported that chemically un-functionalized monolayers of diamond nanoparticles, prepared by the minute detonation synthesis (the so called detonation nanodiamond-DND; Mochalin et al., 2012), promote adhesion and growth of cultured post mitotic hippocampal neurons; emphasizing that chemical surface functionalization does not improve the culture. This conclusion was recently supported also by Edgington et al. (2013). In the context of the potential use of NCD substrates for bidirectional electrical coupling of neurons or muscles with microelectrodes, it is important to note that beside the mechanical, chemical, and biological compatibilities of a given interface, the interfacing material for BMIs should be also electrically tunable. Although both materials (NCD and DND) are of the same chemical composition, 
e.g., carbon in $\mathrm{sp} 3$ bonding configuration with $\mathrm{sp} 2$ inclusions at the grain (NCD) or particle (DND) boundary (Ferrari and Robertson, 2004; Daenen et al., 2009) they differ in the term of technological capabilities. DND do not integrate into a compact layer; they cannot be used to construct flat or three-dimensional electrically conducting tracks. DND could be mainly used in combination with BNCD as a neuron adhesion promoting coating, however, DND can alter surface chemical properties of BNCD, by augmenting the Faradic currents. For example, it has been demonstrated by Holt et al. (2008) that DND particles on gold substrate surface lead to enhancement of redox reactions that can influence cellular processes by promoting the reduction of oxygen with potential production of harmful reactive oxygen species.

Driven by the potential use of NCD for fabrication of in vitro and in vivo bio-electronic platforms, we undertook here the examination of NCD surfaces (rather than DND particles) as substrates for post-mitotic primary vertebrate neurons. In contrast to the expectations based on the literature, we found that it is practically impossible to culture primary neurons, in a defined culture medium, on hydrogen or oxygen terminated NCD. Nevertheless, in view of the physical and chemical advantages of NCD for in vitro and in vivo applications, we have investigated whether chemical functionalization of NCD surfaces can confer permissive substrate properties for adhesion and growth of post mitotic neurons. We report that the problem of bare NCD to promote adhesion and growth of cultured primary neurons can be overcome using conventional poly-D-lysine (PDL)-laminin functionalization. Based on the results, we propose a mechanism that could account for the conflicting results, which on the one hand claim that unfunctionalized NCD provides a permissive substrate for adhesion and growth of cells, while other reports demonstrate the opposite.

\section{MATERIALS AND METHODS NCD FABRICATION}

The diamond films were grown using an Astex AX6550 microwave plasma enhanced chemical vapor deposition system (MWPECVD) working at $\mathrm{H}_{2}(99 \%) / \mathrm{CH}_{4}(1 \%)$ gas mixture and substrate temperature of $700^{\circ} \mathrm{C}$. The substrate was cleaned using standard RCA1 and RCA2 (Radio Corporation America, Kern, 1990) wafer cleaning techniques and subsequently seeded by detonation nanodiamond (DND, $7 \mathrm{~nm}$ size) by spin-coating from an aqueous DND mono-dispersion (NanoAmando ${ }^{\circledR} \mathrm{B}$, NanoCarbo Research Institute, Japan) prior to the deposition. Hydrogen terminated NCD films were achieved by allowing pure hydrogen plasma in the system for $30 \mathrm{~min}$ after stopping the $\mathrm{CH}_{4}$ flow and subsequently cooling in hydrogen. UV ozone treatment for $30 \mathrm{~min}$ (Novascan, PSD digital Ozone system) was used on hydrogen terminated films to give NCD films anoxygen terminated surface. Surface termination was confirmed by XPS and the contact angle measurement, showing hydrophobic surfaces for $\mathrm{H}$-terminated films with the contact angle of $15^{\circ}$ and of $90-92^{\circ}$ for O-terminated NCD films.

\section{SURFACE FUNCTIONALIZATION}

Glass or NCD chips were washed and sterilized by incubation in ethanol $75 \%$ for $2 \mathrm{~h}$. Thereafter the ethanol was thoroughly rinsed with double distilled water and then functionalized by $0.1 \mathrm{mg} \backslash \mathrm{ml}$ PDL (Sigma-Aldrich) and $25 \mu \mathrm{g} \backslash \mathrm{ml}$ laminin (Sigma-Aldrich) in sodium borate solution for $12 \mathrm{~h}$ prior to cells seeding.

\section{CELL CULTURE}

Rat hippocampal neurons were obtained either from one day old new born rats or from 17 days old embryos, as described by Kaech and Banker (2006). Briefly, for embryonic hippocampal cultures the pregnant female was anesthetized, the embryos removed and decapitated. The embryonic or new born hippocampus were removed and treated with papain for $45 \mathrm{~min}$ (Sigma-Aldrich), and serially triturated. Cell density at plating was 250,000-500,000 cells $/ \mathrm{ml}$. Cells were seeded in attachment $\backslash$ seeding medium [Neurobasal medium, 5\% FBS, 2\% B27, 1\%GlutaMAX (All from Life technologies), 1\% Penicillin-Streptomycin Amphotericin B Solution (Biological Industries) ]. $24 \mathrm{~h}$ (1 DIV) after culturing the seeding medium was replaced with serum-free maintenancelfeeding medium (Neurobasal medium, 2\% B27, 1\% GlutaMAX, 1\% Penicillin-Streptomycin Amphotericin B Solution). At 3 DIV $5 \mu \mathrm{M}$ ara-c (Sigma-Aldrich) was added to prevent glial cell's proliferation. Half of the maintenance medium was replaced every 3-5 days by astroglial conditioned medium. Hippocampal cultured cells were kept at $37^{\circ} \mathrm{C}$ in a humidified atmosphere of $5 \% \mathrm{CO}_{2}$. Cultures were kept till 7-21 DIV. All procedures were approved by the Committee for Animal Experimentation at the Institute of Life Sciences of the Hebrew University of Jerusalem.

\section{IMMUNOHISTOCHEMISTRY}

Cultured hippocampal cells were immunolabeled as previously described (Fendyur et al., 2011). Briefly, samples were fixed by 4\% paraformaldehyde (Sigma-Aldrich) in Hank's Balanced Salt Solution (HBSS, Biological Industries) for $30 \mathrm{~min}$, washed with HBSS before membrane permeabilization with $0.1 \%$ TritonX-100 (BDH Chemicals) in HBSS for $30 \mathrm{~min}$. After washes with Tween $0.1 \%$ (J.T.Baker) in HBSS, cells were incubated for $1 \mathrm{~h}$ in blocking solution [BS, 2\% chicken albumin (Sigma-Aldrich) in Tween 0.1\%]. Then samples were incubated with primary antibodies in $1 \% \mathrm{BS}$, overnight at $4^{\circ} \mathrm{C}$ : neurons were labeled for neuron-specific intermediate filaments with mouse anti neurofilament antibodies; Glial cells were labeled for glial fibrillary acidic protein (GFAP) with primary anti-GFAP rabbit monoclonal antibodies. The next day the samples were washed repeatedly with $0.1 \%$ Tween and incubated with secondary antibodies in 1\% BS for $1 \mathrm{~h}$ : goat anti-mouse secondary antibodies conjugated to Cy2 (Jackson ImmunoResearch Laboratories, Inc), and goat anti-rabbit secondary antibodies conjugated to Cy3 (Life technology). Cells were counterstained with the nuclear marker DAPI (Sigma-Aldrich) for $1 \mathrm{~h}$, at room temperature. Samples were washed with $\mathrm{HBSS}$, and stored at $4^{\circ} \mathrm{C}$ in anti-fade n-propyl gallate (Sigma-Aldrich) solution in 50\% glycerol till imaging. Confocal imaging of the immunolabeled cultures was done using D-Eclipse C1 imaging system (Nikon) mounted on an Eclipse TE-2000 microscope (Nikon). Images were collected and processed using EZ-C1 software (Nikon). Scanning was done in sequential mode: red was excited with $543 \mathrm{~nm} \mathrm{He}-$ Ne laser and collected with $605 \pm 75$ band pass filter; green was excited with $488 \mathrm{~nm}$ Argon laser and collected with $515 \pm 30$ band pass filter; blue excited with $405 \mathrm{~nm}$ Diode and collected with 

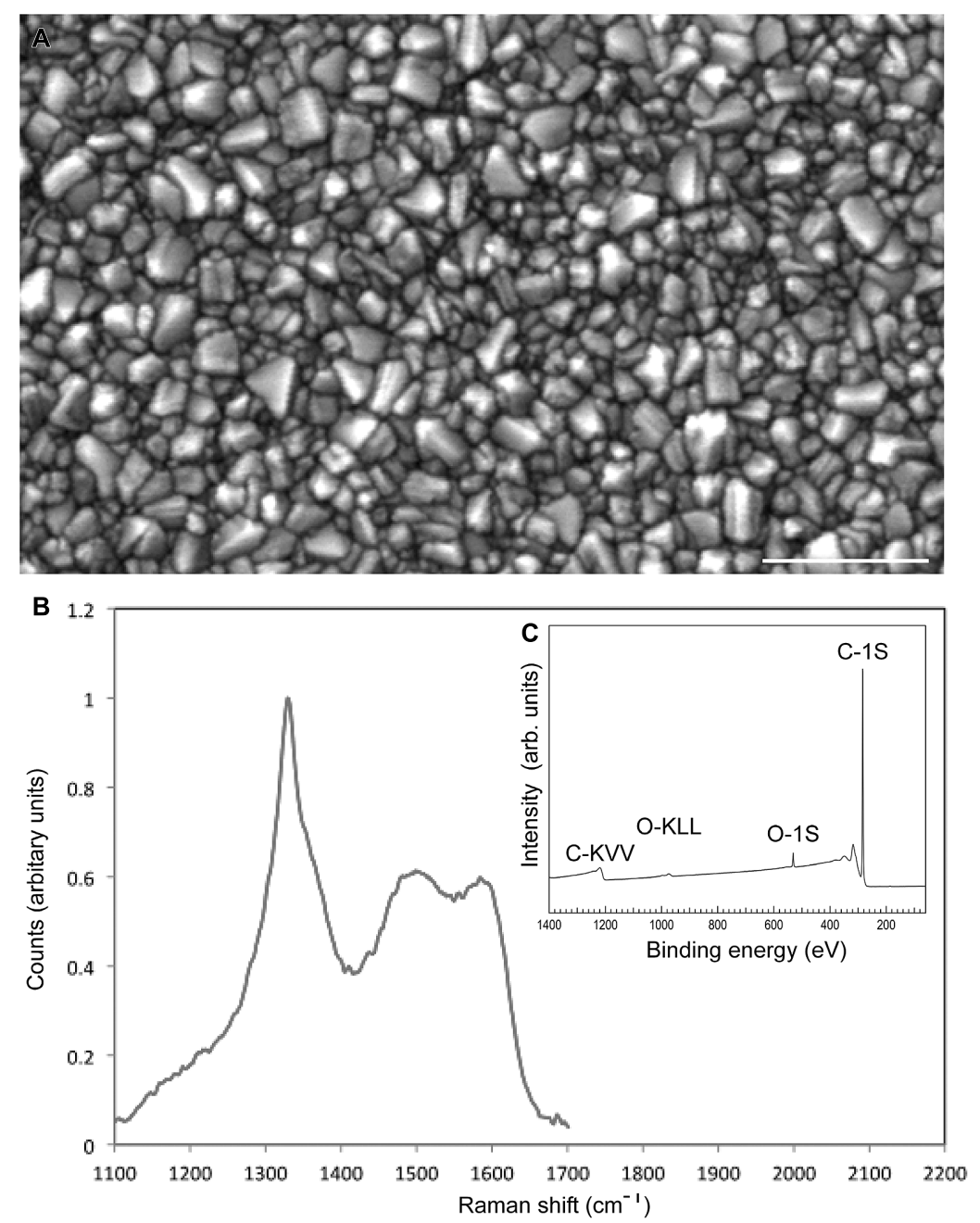

FIGURE 1 | Characterization of the nanocrystalline surface. (A) SEM image of the nanocrystalline diamond (NCD) film morphology with Rmns $\sim 17 \mathrm{~nm}$. (B) The Raman spectra of the NCD film showing the zone-center phonon $\mathrm{sp}^{3}$ diamond line at $1332 \mathrm{~cm}^{-1}$ and a higher wave number signal, consisting of disordered and $\mathrm{sp}^{2}$ bonded formed at diamond gain boundaries. Content of non-diamond carbon is estimated to be $0.7 \%$. (C) shows an XPS spectrum of oxidized NCD surface. Bar in $\mathrm{A}=0.5 \mu \mathrm{m}$.
$450 \pm 35$ band pass filter. Images were prepared using the opensource image analysis program Image (NIH, USA) and Photoshop CS6.

\section{CALCIUM IMAGING AND FORMATION OF A CONNECTIVITY MAP}

Ten to 14-day-old cultures were incubated for $45 \mathrm{~min}$ in a $37^{\circ} \mathrm{C}$ incubator in a $5 \mu \mathrm{M}$ fluo-4 (AM; Molecular Probes) dissolved in HBSS. The fluo-4 AM solution was washed away with HBSS and the culture was then incubated in fluo-4 free medium for 15 min. Cells were imaged with a $20 \times$ objective $(\mathrm{NA}=0.75)$ using the confocal microscope system described above. Seconds long stimulation of a single neuron at the center of the field of view was applied with a fire polished patch electrode and the response imaged by the frame-scan modes. The second long stimulation led to a buildup of intracellular calcium levels that could be detected by the low NA objective and the slow laser scan used ( $3.9 \mathrm{~s} /$ frame). Sequential confocal fluo- 4 images were used to generate a neuronal connectivity map of a relatively large field of view. Upon cessation of the stimulation the fluorescent signal recovered to control level.

The connectivity map (Figure 7) was generated by subtracting each image from the previous one. Eight consecutive subtracted frames, from the stimulation onset, were then sequentially color coded in accordance to a lookup color table. The total number of cell bodies activated by the stimulation was counted and the largest distance between the stimulated cell and the follower cells within the field of view was measured.

\section{SCANNING ELECTRON MICROSCOPY}

For SEM analysis cells cultured on the NCD or glass substrate were fixed, dehydrated, within the culturing dish as previously described (Spira et al., 2003). Briefly, hippocampal primary cultured cells were fixed by $3 \%$ glutaraldehyde (Electron Microscopy Science) in cacodylate buffer (Agar Scientific, Stansted) at pH7.4. The cells were then washed in cold $0.1 \mathrm{M}$ cacodylate buffer, pH7.4. The cells 


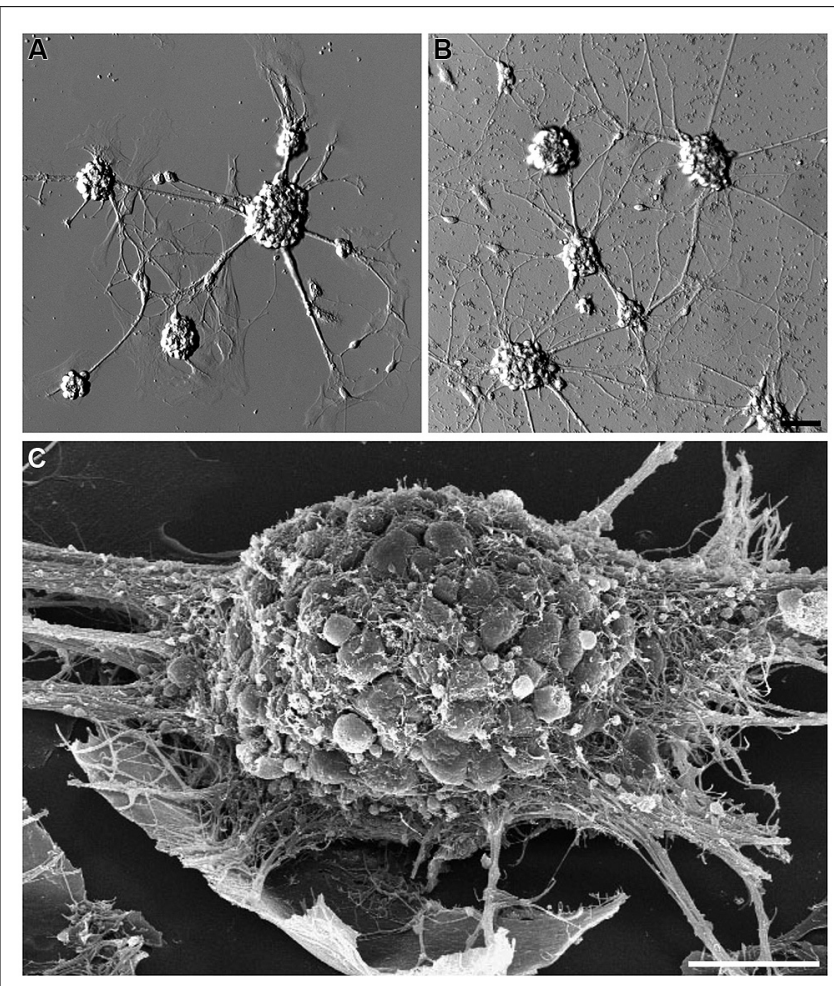

FIGURE 2 | Clusters of hippocampal neurons cultured on bare glass or NCD surfaces. Embryonic (E17) hippocampal neurons cultured on bare glass (A) and bare NCD (B), 3 DIV; bar $=50 \mu \mathrm{m}$. (C) SEM image of hippocampal neurons cultured on bare glass, 7 DIV; bar $=20 \mu \mathrm{m}$.

were post-fixed in 1\% osmium tetroxide (Electron Microscopy Science) and $1.5 \% \mathrm{~K}_{3} \mathrm{Fe}(\mathrm{CN})_{6}$ (Sigma-Aldrich). Dehydration was carried out through a series of ethanol solutions and washed two times for $30 \mathrm{~min}$ with fresh 100\% EtOH before critical point drying with liquid $\mathrm{CO}_{2}$ in a SAMDRI-PVT-3D (Tousimis, USA). Once dry the samples were sputtered with gold in an SPI-Module ${ }^{\text {TM }}$ Sputter Coater Module (SPI Supplies, USA). Images were taken with an Extra High Resolution Scanning Electron Microscopy MagellanTM 400L using an accelerating voltage of $5 \mathrm{kV}$.

\section{STATISTICAL ANALYSIS}

The averaged density of cells adhering to PDL functionalized control glass or NCD substrates were calculated by averaging the densities (cells number/standard surface area of $607 \times 607 \mu \mathrm{m}$ ) obtained from five randomly selected areas on days 3,7 , and 10 in culture (DIV). The data are presented as mean \pm standard deviation. $t$-tests was performed using Excel software.

\section{RESULTS}

\section{SYNTHESIS OF NCD FILMS}

For the present study, $150 \mathrm{~nm}$ thick high quality NCD films were synthesized on fused silica substrate $(10 \mathrm{~mm} \times 5 \mathrm{~mm} \times 0.5 \mathrm{~mm}$ size). Substrates were seeded in an aqueous suspension of DND particles to promote the diamond crystal nucleation and subsequently to initiate diamond layer growth (Barros et al., 2011). NCD films were grown in a resonance cavity microwave plasma

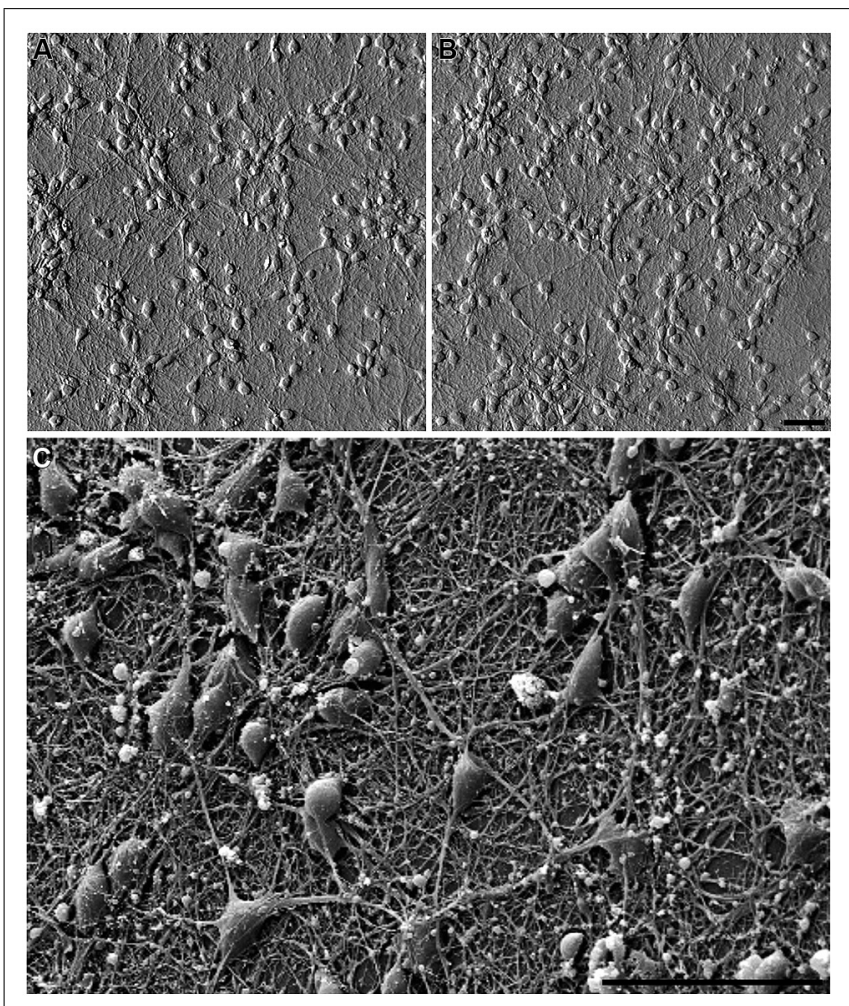

FIGURE 3 | Embryonic hippocampal neurons (7 DIV) cultured on:

(A) PDL-laminin functionalized glass and (B) PDL-laminin functionalized

NCD. No difference in adhesion and growth is seen on the two substrates; bar $=50 \mu \mathrm{m}$. (C) SEM image of embryonic hippocampal neurons cultured on PDL-laminin functionalized NCD. Note the dense growth of neuritis; bar $=50 \mu \mathrm{m}$.

enhanced chemical vapor deposition system (MW-PECVD) using $1 \% \mathrm{CH}_{4}$ mixture in hydrogen at substrate temperature of $700^{\circ} \mathrm{C}$ (see Experimental methods for details: Williams et al., 2008; Daenen et al., 2009). The morphology of NCD films was composed of well-developed faceted nano-crystals of random orientation and is shown in Figure 1A. Figure 1B shows the Raman spectra of NCD films. The sp2 and disordered carbon originating at the grain boundaries, as typical from NCD films, was as low as $0.7 \%$ as determined by the numerical fitting of Raman spectra (for detail Ferrari and Robertson, 2004). The inset of Figure 1C shows an XPS survey scan of an oxidized NCD surface, acquired immediately after the MW-PECVD preparation and oxidizing the NCD diamond film in Ozone. Clearly, the spectrum displays ultra-clean diamond surface dominated only by the emission from carbon and oxygen. The C-1s core level (binding energy $284 \mathrm{eV}$ ) and in addition to C-lines, an O-1s signal are visible $(531 \mathrm{eV})$ which can be assigned to the oxygen-terminated surface. Such surfaces were further used for neuron adhesion study.

\section{INCOMPATIBILITY OF NCD SUBSTRATE FOR PRIMARY NEURON ADHESION AND GROWTH}

We tested the compatibility of bare NCD surface for adhesion and growth by culturing primary embryonic (E17) or newborn rat 

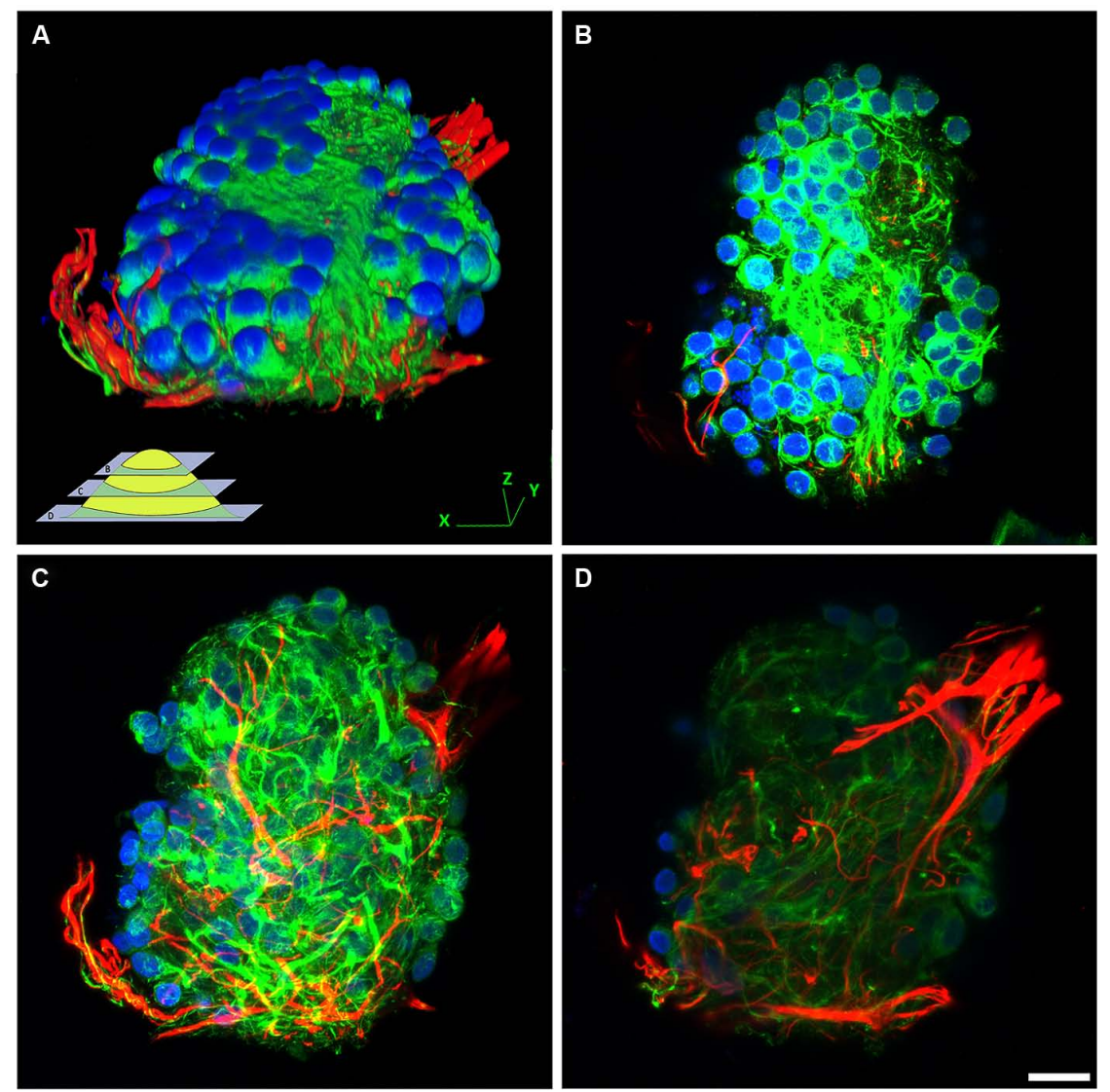

FIGURE 4 |The structural organization of cell-aggregates formed on bare NCD substrate. Confocal image of immunolabeled (green- NF, redGFAP, blue- DAPI) hippocampal neurons and glia cultured on bare NCD, 10 DIV. (A) Three dimensional reconstructed view of a cell-cluster. The insert illustrate the focal planes of the images shown in $\mathbf{B}, \mathbf{C}$, and $\mathbf{D}$
(B) Top layer of the cell cluster (a focal slice of $18 \mu \mathrm{m}$ ), consists mainly of neuronal cell bodies. (C) Middle part of the cell-cluster (thickness of $-19.5 \mu \mathrm{m}$ ), consists of neurites. (D) Bottom part of the cell cluster (slice thickness of $-12.5 \mu \mathrm{m}$ ), close to the substrate level, showing glial processes; bar $=20 \mu \mathrm{m}$. hippocampal cells on NCD, with hydrogen-(H-hydrophobic) and oxygen (O-hydrophilic)-termination. As a control to these experiments, we cultured neurons in the same experimental sessions on

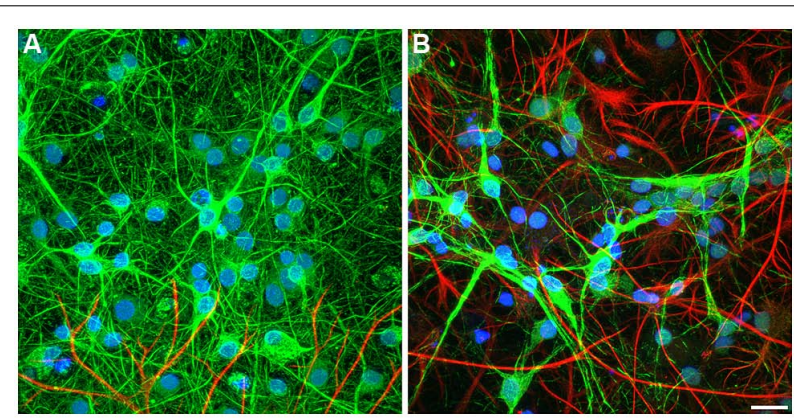

FIGURE 5 | Confocal image of immunolabeled hippocampal cells. (A) embryonic, (B) newborn neurons cultured on PDL-laminin functionalized NCD, 10 DIV. The embryonic culture consists of mainly of neurons whereas newborns culture conssit of mixed neuron-glia cells. Both embryonic and new born cultures survive on functionalized NCD for over 3 weeks; bar $=20 \mu \mathrm{m}$. bare- and PDL-laminin functionalized glass substrates. The criteria used for comparing the cultures qualities were: adhesion of the cells to the substrate, the development of neurites on the substrate, survival time of the culture, and neuronal network functioning as revealed by calcium imaging of evoked network activity.

Hippocampal cells (neurons and glia) grown on bare NCD, or on bare glass substrates revealed poor adhesion and cell clustering (Figure 2). The cell clusters were interconnected by a small number of extensions. This is in contrast to control cultures of embryonic or new born hippocampal cells grown on PDL-Laminin functionalized glass-substrate which adhered well to the substrate and generated an elaborate network of neuritis (Figure 3A). The self-assembly of cells into clusters, rather than adhesion of individual cells to the substrate, reduces the contact surface area between the cells and the substrate, and enhances cell-cell contact. Such self-assembly of aggregates reflects significant adhesion-incompatibility between the cells and the substrate relative to the adhesion among cells. Three dimensional reconstructions of confocal microscope optical sections obtained at $0.25 \mu \mathrm{m}$ steps revealed that in newborn cultures (that are rich with glia) the cells that interface with the substrate are mainly 


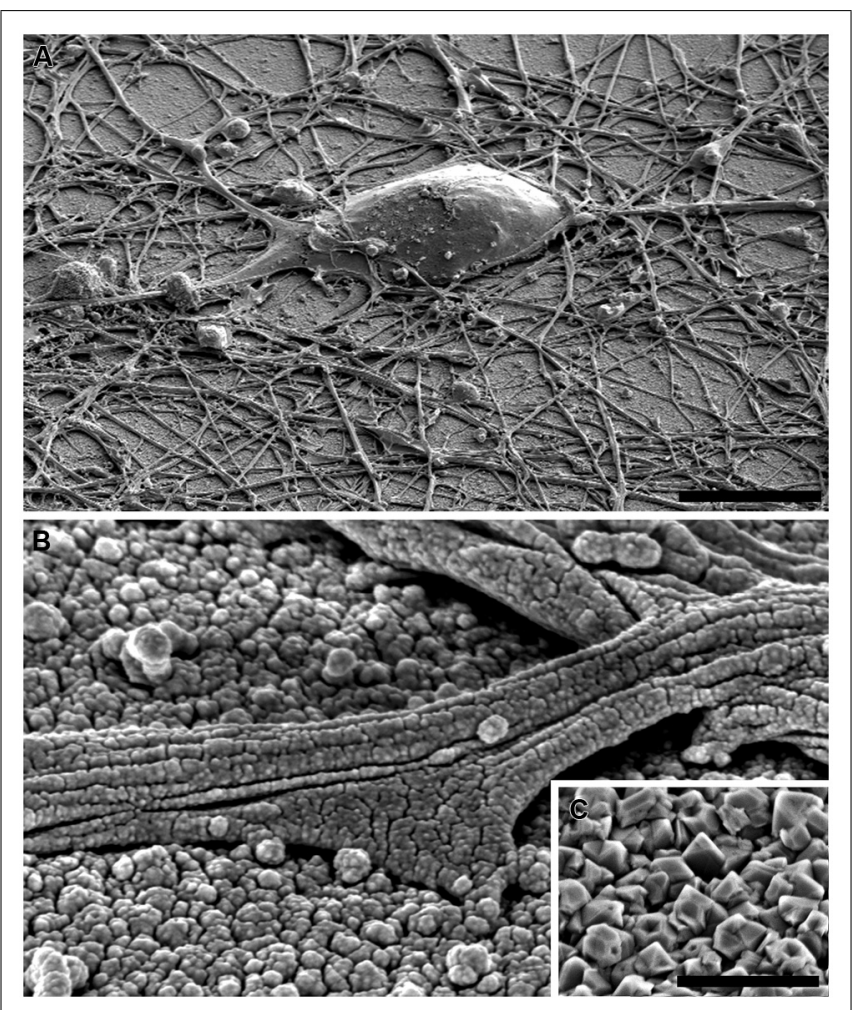

FIGURE 6 | SEM image of embryonic hippocampal neurons cultured on PDL-laminin functionalized NCD, 7 DIV. (A) low magnification of a neuron; bar $=10 \mu \mathrm{m}$. (B) a neurite adhering to the ND layer. (C) NCD imaged without gold spattering; bar $=500 \mathrm{~nm}$.

glia, whereas the neuronal cell bodies occupy the upper layers facing the bathing solution (Figure 4). Cultures prepared from embryos (E17) are almost glia-free, nevertheless they also form aggregates. These clusters detach from the substrate and degenerate within a number of days. No significant differences in cell-substrate adhesion were noticed among hippocampal cells cultured on bare glass substrate and the various NCD types used.

We conclude that post mitotic primary hippocampal neurons and glia grown on un-functionalized NCD or glass form cells aggregates as they do not adhere well to the substrate.

\section{HIPPOCAMPAL CELL ADHESION AND GROWTH ON FUNCTIONALIZED NCD SUBSTRATES}

We next examined whether chemical functionalization of NCD surface solves the problem of cell-adhesion incompatibility to NCD substrates. To that end we employed the classical and robust PDL-laminin glass surface coating procedure. We found that functionalization of NCD substrates by PDL-laminin generates an adhesive and permissive substrate for primary neurons culturing (Figures 3, 5 and 6). Under these conditions, both embryonic and new born hippocampal cells adhere and extend a dense network of neuritis on the substrate. No significant differences in adhesion and survival over the time of observations were noted between neurons grown on functionalized glass or the various NCD substrates using morphological criteria (Figure 3) and functional assessments (see Neuronal Network Activity on Functionalized NCD Substrates). Thus, for cultures prepared from 17 days old embryos: on DIV 3 the averaged density of neurons on a standard area of functionalized glass (number of counted areas $n=5$ ) was $683 \pm 94$ for control and on functionalized NCD $718 \pm 173$. These values are not significantly different $(\alpha=0.05, p=0.7)$. Likewise, the cell densities on DIV 7 and 10 did not change overtime on both the functionalized glass and NCD substrates exhibiting on DIV 7 densities of $528 \pm 123$ in control and $496 \pm 151$ on functionalized NCD $(p=0.7)$, and on DIV $10527 \pm 151$ in control and $630 \pm 137$ on functionalized NCD $(p=0.29)$. The density of cultured cells (neurons and astrocytes) prepared from new born rats did not reveal any differences between the functionalized glass and NCD substrate but revealed a significant increase in the number of astrocytes on day 10 of the culture. Thus, on DIV 3 the averaged density of cells in the control was $247 \pm 14$ and on NCD $273 \pm 105$ ( $\alpha=0.05, p=0.6$ ), on DIV 7 in control the average was $177 \pm 15$ and on functionalized NCD $229 \pm 46(p=0.01)$. On DIV 10 of the cultures prepared from new borne rats a large and significant increase in the density of the cells was observer on both glass and NCD substrates. The averaged density on glass was $437 \pm 46$ and on NCD $524 \pm 153(p=0.26)$. The significant increase in the number of cells from DIV 7 to 10 is due to glia cell proliferation.

\section{NEURONAL NETWORK ACTIVITY ON FUNCTIONALIZED NCD SUBSTRATES}

The physical, chemical, and morphological nature of the substrates to which cells adhere and on which cells develop may have significant effects on cells morphology, their biophysical properties (electroanatomy) and, in the case of neurons, their synaptic functions and the overall network activities [for example (Fabbro etal., 2013)]. To assess whether neurons grown on NCD functionalized with PDL-Laminin maintain excitable membrane properties that promote propagation of action potentials along neurites, form functional connectivity that permit polysynaptic communication, we imaged the fluorescent signals generated by the calcium indicator fluo-4 in response to a long lasting train of stimuli delivered to a single neuron at the center of the field of view. To that end, the neurons were loaded with acetoxymethyl (AM) ester fluo-4 and then after removal of the excess fluo4-AM and incubation period to allow hydrolyze of the ester, a neuron located at the center of field was stimulated by a fire polished microelectrode. Live confocal imaging of the fluo-4 fluorescent revealed the propagation of the fluorescent signal from the stimulated cell to neighboring cell bodies along neurites (Figure 7). Analysis of the number of neurons activated by the stimulation in control (neurons cultured on functionalized glass substrate) and neurons grown on functionalized NCD were similar $(45 \pm 13.55$ in control and $61.17 \pm 41.04$ on the NCD substrate. $t$-test for unequal variances $n=5$ and 6 , respectively, is for $\alpha=0.05, p=0.42$ ). The maximal distance of the fluorescent signals propagation were the same ranging between 254 and $423 \mu \mathrm{m}$ (322 \pm 75 in control and $317 \pm 66$ on the NCD substrate. $t$-test for unequal variances $n=5$ and 


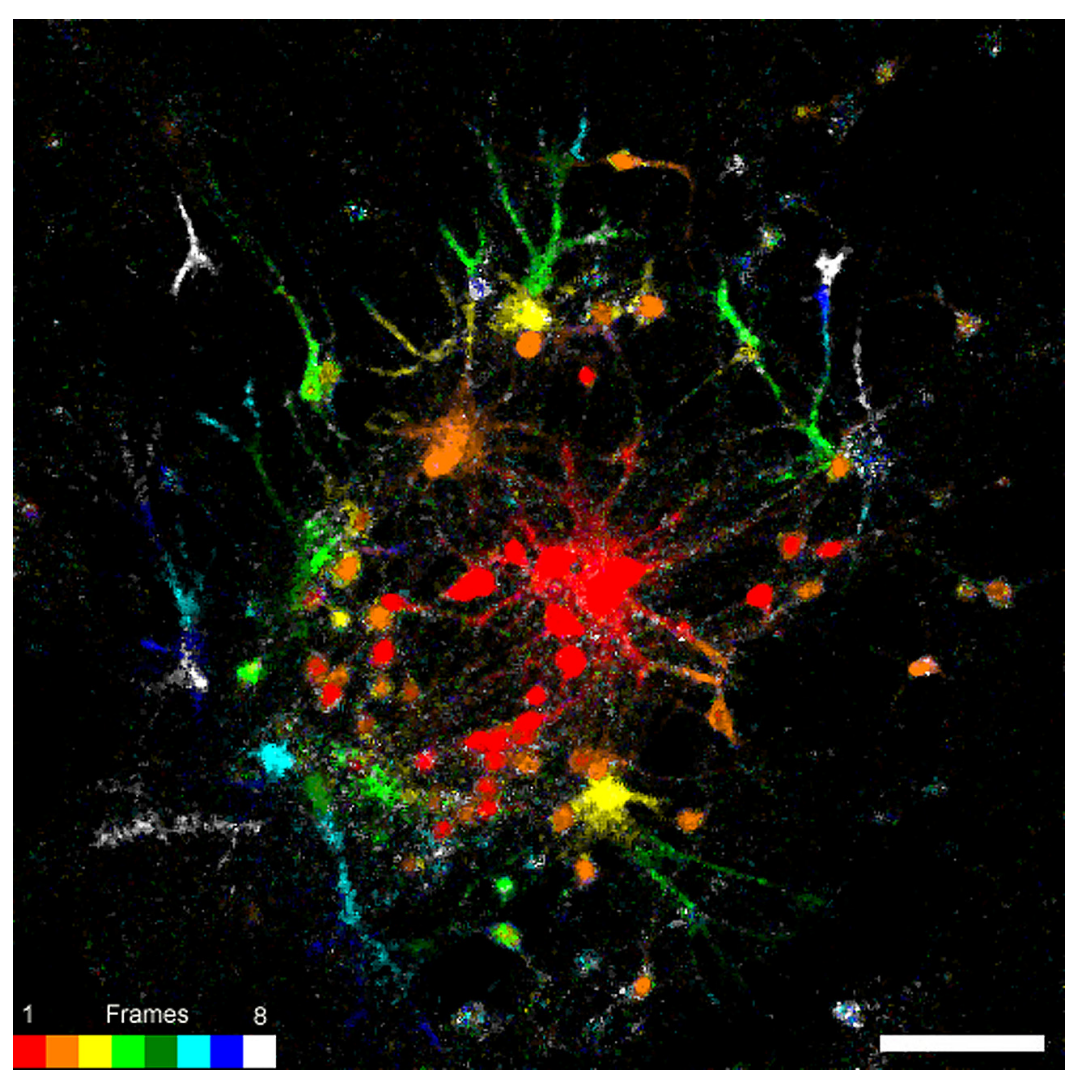

FIGURE 7 | Connectivity map of 14 days old cultured embryonic neurons on PDL-laminin functionalized NCD surface. Nine consecutive confocal images were grabbed. The first before the onset of stimulation and the rest during and after the stimulation. Consequent images were subtracted (image $n+1$ - image $n$ ) to generate eight subtracted images. The resulting images were color coded accordance to the lookup $r$ table attached to the figure. Note that the fluo-4 fluorescent signals propagated radially (from red to white along the pseudo color lookup table). The average number of cells that were excited by stimulation of a single neuron and the maximal distance of signal propagation was similar in cultures grown on functionalized glass and NCD. Bar $=20 \mu \mathrm{m}$.
6 , respectively, is for $\alpha=0.005, p=0.91$ ). We conclude that the overall network connectivity of cells grown on functionalized NCD is similar to those grown on functionalized glass.

\section{DISCUSSION}

The main observations of the present study is that in contrast to the general impression generated by earlier studies we found that post-mitotic primary rat hippocampal neurons and glial cells do not adhere and develop when cultured in defined medium on bare, oxidized and H-terminated NCD substrates. Nevertheless, classical chemical functionalization of the NCD substrate by PDL-laminin renders the substrate with permissive growth properties for these cells. Under these culturing conditions primary hippocampal neurons develop excitable membrane properties and excitatory synapses that communicate among them.

The incompatibility of bare NCD as a substrate for primary neuron culture reported here is in contradiction with earlier studies reporting that cell lines as well as hippocampal neurons adhere and develop on various NCD substrates without chemical surface functionalization prior to cell seeding. We devote the following paragraph to briefly propose a possible mechanism to account for this contradiction.

Under in vivo conditions cells are attached to each other by extracellular matrix (ECM) and cell adhesion molecules (CAMs; Abedin and King, 2010). Whereas the ECM and the CAM are secreted and expressed (respectively) by all cell types derived from multicellular organisms, their molecular, biochemical nature and levels of expression differ in different cell types and under different physiological conditions (for example see Barros etal., 2011). These differences are so substantial that in vitro cell culturing methods take advantage of the differences to isolate certain cell types from others. This is done, for example, by seeding a heterogeneous cell suspension on a common un-functionalized glass or plastic substrates. Under these conditions some cell types adhere to the bare substrate while others remain in suspension for a longer period of time (see for Danoviz and Yablonka-Reuveni, 2012). The selective cellsadhesivity reflects differences in the nature and rates of ECM secretion and CAM expression. The non-adhering or the adhering cells can then be collected. Differences in ECM molecular nature and secretion are also observed under in vivo conditions. For example regrowth of peripheral axons depend to 
a large extent on ECM secreted by supporting Schwann cells (Gonzalez-Perez et al., 2013). For these reasons, most classical and contemporary primary neuron culturing-procedures heavily rely on conscious chemical functionalization of the substrates prior to cells seeding.

It is conceivable that cell lines and primary proliferating cell types that are characterized by effective ECM secretion adhere and divide when plated on chemically un-functionalized surfaces. On the other hand, generations of cell biologists have established that in vitro culturing of primary neurons on glass substrate require surface functionalization prior to seeding. Therefore the reports of Thalhammer et al. (2010) and Edgington et al. (2013) who demonstrated that primary neurons which do not adhere to NCD adhere and grow on non-functionalized DND are somewhat surprising. The adhesion to DND could be explained by assuming that in these studies the DND surface was unconsciously functionalized by serum protein during the incubation step of the substrate in the seeding medium which contained 10\% FBS. Interestingly, the protocol used by us also involved a $\sim 20 \mathrm{~h}$ incubation period in 5\% FBS. Nevertheless, as evident by our study, this and even extended periods of incubation, were insufficient to confer adhesive properties to our NCD substrate. The neurons did not adhere to $\mathrm{sp} 3$ carbon reach NCD grains neither to sp2 carbon rich grain boundaries. Both oxygen terminated (negative surface charge) or $\mathrm{H}$-terminated bare NCD surfaces (positive surface charge) yielded to same negative result.

A possible mechanism that could account for this phenomenon could be related to differences in protein absorption capacities of DND and NCD films as the DND used by Thalhammer et al. (2010) and Edgington et al. (2013) are composed of round diamond particles of diameter $\sim 8-10 \mathrm{~nm}$ while NCD films used in this study are composed of faceted crystals with Rms $\sim 15-17 \mathrm{~nm}$.

In conclusion, we demonstrate that bare NCD based substrates are not providing permissive adhesion and growth substrate for cultured primary neurons and glia. The literature demonstrates that cell lines and other proliferating cells can adhere and proliferate on ND substrates relying on their innate ECM resources. In contrast, cells that do not secret effective ECM (as primary neurons) will not adhere to NCD substrate and degenerate. We conclude that, whereas bare NCD has a broad range of advantageous material properties unusual cell adhesion properties are not included among them. Nevertheless, simple functionalization strategies make NCD a permissive substrate for adhesion and growth of post mitotic primary neurons. As functionalized NCD surfaces supports well neural adhesion its unique electrical mechanical and chemical properties is a highly attractive material for construction of in vitro and in vivo MEAs and BMIs.

\section{ACKNOWLDEGMENTS}

The study was supported by a grant from the European commission FP7-NMP SL-280778-2 (MERIDIAN). Silviya M. Ojovan and Mathew McDonald were supported by the EC-FP7 Marie Curie ITN PITN-GA-264872 (NAMASEN). Part of the study was carried out at the Smith family and Prof. Elkes laboratory for collaborative research.

\section{REFERENCES}

Abedin, M., and King, N. (2010). Diverse evolutionary paths to cell adhesion. Trends Cell Biol. 20, 734-742. doi: 10.1016/j.tcb.2010.08.002

Amaral, M., Gomes, P. S., Lopes, M. A., Santos, J. D., Silva, R. F., and Fernandes, M. H. (2009). Cytotoxicity evaluation of nanocrystalline diamond coatings by fibroblast cell cultures. Acta Biomater. 5, 755-763. doi: 10.1016/j.actbio.2008.08.015

Ariano, P., Budnyk, O., Dalmazzo, S., Lovisolo, D., Manfredotti, C., Rivolo, P., et al. (2009). On diamond surface properties and interactions with neurons. Eur. Phys. J. E Soft Matter 30, 149-156. doi: 10.1140/epje/i2009-10520-9

Barros, C. S., Franco, S. J., and Muller, U. (2011). Extracellular matrix: functions in the nervous system. Cold Spring Harb. Perspect. Biol. 3, a005108. doi: 10.1101/cshperspect.a005108

Daenen, M., Zhang, L., Erni, R., Williams, O. A., Hardy, A., Van Bael, M. K., et al. (2009). Diamond nucleation by carbon transport from buried nanodiamond TiO2 sol-gel composites. Adv. Mat. 21, 670-673. doi: 10.1002/adma.200802305

Danoviz, M. E., and Yablonka-Reuveni, Z. (2012). Skeletal muscle satellite cells: background and methods for isolation and analysis in a primary culture system. Methods Mol. Biol. 798, 21-52. doi: 10.1007/978-1-61779343-1_2

Edgington, R. J., Thalhammer, A., Welch, J. O., Bongrain, A., Bergonzo, P., Scorsone, E., et al. (2013). Patterned neuronal networks using nanodiamonds and the effect of varying nanodiamond properties on neuronal adhesion and outgrowth. $J$. Neural Eng. 10:056022. doi: 10.1088/1741-2560/10/5/056022

Fabbro, A., Prato, M., and Ballerini, L. (2013). Carbon nanotubes in neuroregeneration and repair. Adv. Drug Deliv. Rev. 65, 2034-2044. doi: 10.1016/j.addr.2013.07.002

Fendyur, A., Mazurski, N., Shappir, J., and Spira, M. E. (2011). Formation of essential ultrastructural interface between cultured hippocampal cells and gold mushroom-shaped MEA- toward "IN-CELL" recordings from vertebrate neurons. Front. Neuroeng. 4:14. doi: 10.3389/fneng.2011.00014

Fendrych, F., Taylor, A., Peksa, L., Kratochvilova, I., Vlcek, J., Rezacova, V., et al. (2010). Growth and characterization of nanodiamond layers prepared using the plasma-enhanced linear antennas microwave CVD system. J. Phys. D Appl. Phys. 43, 374018. doi: 10.1088/0022-3727/43/37/374018

Ferrari, A. C., and Robertson, J. (2004). Raman spectroscopy of amorphous, nanostructured, diamond-like carbon, and nanodiamond. Philos. Trans. R. Soc. Lond A 362, 2477-2512. doi: 10.1098/rsta.2004.1452

Ganesan, K., Garrett, D. J., Ahnood, A., Shivdasani, M. N., Tong, W., Turnley, A. M., et al. (2014). An all-diamond, hermetic electrical feedthrough array for a retinal prosthesis. Biomaterials 35, 908-915. doi: 10.1016/j.biomaterials.2013.10.040

Gonzalez-Perez, F., Udina, E., and Navarro, X. (2013). Extracellular matrix components in peripheral nerve regeneration. Int. Rev. Neurobiol. 108, 257-275. doi: 10.1016/B978-0-12-410499-0.00010-1

Hadjinicolaou, A. E., Leung, R. T., Garrett, D. J., Ganesan, K., Fox, K., Nayagam, D. A., et al. (2012). Electrical stimulation of retinal ganglion cells with diamond and the development of an all diamond retinal prosthesis. Biomaterials 33, 5812-5820. doi: 10.1016/j.biomaterials.2012.04.063.

Holt, K. B., Ziegler, C., Caruana, D. J., Zang, J., Millan-Barrios, E. J., Hu, J., et al. (2008). Redox properties of undoped $5 \mathrm{~nm}$ diamond nanoparticles. Phys. Chem. Chem. Phys. 10, 303-310. doi: 10.1039/B711049a

Kaech, S., and Banker, G. (2006). Culturing hippocampal neurons. Nat. Protoc. 1, 2406-2415. doi: 10.1038/nprot.2006.356

Kern, W. (1990). The evolution of silicon-wafer cleaning technology. J. Electrochem. Soc. 137, 1887-1892. doi: 10.1149/1.2086825

Klauser, F., Hermann, M., Steinmuller-Nethl, D., Eiter, O., Pasquarelli, A., Bertel, E., et al. (2010). Direct and protein-mediated cell attachment on differently terminated nanocrystalline diamond. Chem. Vap. Deposition 16, 42-49. doi: $10.1002 /$ cvde. 200906828

Mochalin, V. N., Shenderova, O., Ho, D., and Gogotsi, Y. (2012). The properties and applications of nanodiamonds. Nat. Nanotech. 7, 11-23. doi: 10.1038/Nnano.2011.209

Singh, Y. S., Sawarynski, L. E., Michael, H. M., Ferrell, R. E., Murphey-Corb, M. A., Swain, G. M., et al. (2010). Boron-doped diamond microelectrodes reveal reduced serotonin uptake rates in lymphocytes from adult rhesus monkeys carrying the short allele of the 5-HTTLPR. Acs Chem. Neurosci. 1, 49-64. doi: $10.1021 / \mathrm{Cn} 900012 \mathrm{y}$

Spira, M. E., Oren, R., Dormann, A., and Gitler, D. (2003). Critical calpaindependent ultrastructural alterations underlie the transformation of an axonal 
segment into a growth cone after axotomy of cultured Aplysia neurons. J. Comp. Neurol. 457, 293-312. doi: 10.1002/cne.10569

Thalhammer, A., Edgington, R. J., Cingolani, L. A., Schoepfer, R., and Jackman, R. B. (2010). The use of nanodiamond monolayer coatings to promote the formation of functional neuronal networks. Biomaterials 31, 2097-2104. doi: 10.1016/j.biomaterials.2009.11.109

Williams, O. A., Nesladek, M., Daenen, M., Michaelson, S., Hoffman, A., Osawa, E., et al. (2008). Growth, electronic properties and applications of nanodiamond. Diam. Relat. Mater. 17, 1080-1088. doi: 10.1016/j.diamond.2008.01.103

Xiao, X. C., Wang, J., Liu, C., Carlisle, J. A., Mech, B., Greenberg, R., et al. (2006). In vitro and in vivo evaluation of ultrananocrystalline diamond for coating of implantable retinal microchips. J. Biomed. Mater. Res. B Appl. Biomater. 77B, 273-281. doi: 10.1002/Jbm.B.30448

Zivcova, Z. V., Frank, O., Petrak, V., Tarabkova, H., Vacik, J., Nesladek, M., et al. (2013). Electrochemistry and in situ Raman spectroelectrochemistry of low and high quality boron doped diamond layers in aqueous electrolyte solution. Electrochim. Acta 87, 518-525. doi: 10.1016/j.electacta.2012.09.031
Conflict of Interest Statement: The authors declare that the research was conducted in the absence of any commercial or financial relationships that could be construed as a potential conflict of interest.

Received: 11 April 2014; accepted: 23 May 2014; published online: 11 June 2014. Citation: Ojovan SM, McDonald M, Rabieh N, Shmuel N, Erez H, Nesladek M and Spira ME (2014) Nanocrystalline diamond surfaces for adhesion and growth of primary neurons, conflicting results and rational explanation. Front. Neuroeng. 7:17. doi: 10.3389/fneng.2014.00017

This article was submitted to the journal Frontiers in Neuroengineering.

Copyright (C) 2014 Ojovan, McDonald, Rabieh, Shmuel, Erez, Nesladek and Spira. This is an open-access article distributed under the terms of the Creative Commons Attribution License (CC BY). The use, distribution or reproduction in other forums is permitted, provided the original author(s) or licensor are credited and that the original publication in this journal is cited, in accordance with accepted academic practice. No use, distribution or reproduction is permitted which does not comply with these terms. 